\title{
Overview of Uncovered and Suspected Large-scale Structures behind the Milky Way
}

\author{
Renée C. Kraan-Korteweg ${ }^{1}$ and Patrick A. Woudt ${ }^{2}$ \\ 1 Depto. de Astronomía, Univ. de Guanajuato, Apartado Postal 144, \\ Guanajuato, GTO 36000, Mexico \\ kraan@astro.ugto.mx \\ 2 ESO, Karl-Schwarzschildstr. 2, D-85748 Garching bei München, Germany \\ pwoudt@eso.org \\ Received 1998 November 2, accepted 1999 February 1
}

\begin{abstract}
Various dynamically important extragalactic large-scale structures in the local Universe lie behind the Milky Way. Most of these structures (predicted and unexpected) have only recently been made 'visible' through dedicated deep surveys at various wavelengths. The wide range of observational searches (optical, near infrared, far infrared, radio and X-ray) for galaxies in the Zone of Avoidance (ZOA) will be reviewed and the uncovered and suspected large-scale structures summarised. Particular emphasis is given to the Great Attractor region where the existence of yet another cluster is suspected (Woudt 1998). Predictions from reconstructions of the density field in the ZOA are discussed and compared with observational evidence. Although no major structures are predicted out to about $v \lesssim 10,000 \mathrm{~km} \mathrm{~s}^{-1}$ for which no observational evidence exists, the comparison between reconstructed density fields and the observed galaxy distribution remain important as they allow derivations of the density and biasing parameters.
\end{abstract}

Keywords: zone of avoidance - surveys - ISM: dust, extinction - large-scale structure of universe

\section{Introduction}

In the last few years, much progress has been achieved in uncovering the galaxy distribution behind the Milky Way through various multi-wavelength approaches. These sometimes quite tedious tasks are necessary in order to understand the dynamics in the nearby Universe and answer the question whether the perturbations on the smooth Hubble expansion, such as the dipole in the Cosmic Microwave Background and other velocity flow fields, can be fully explained by the irregular galaxy distribution, i.e. mass distribution if galaxies are fair tracers of mass. In Section 2, the various observational methods are described and the large-scale structures (LSS) uncovered to date summarised. With respect to $\mathrm{HI}$ and near infrared surveys, only their characteristicsadvantages, limitations and selection effects - in context to other approaches are discussed as specific surveys and results are presented in detail elsewhere in this volume (cf. Henning et al. 1999; Rivers et al. 1999; Juraszek 1999; Schröder et al. 1999). In the last section, theoretical predictions applied to the recent deeper sampled galaxy surveys covering volumes out to $v \leq 10000 \mathrm{~s}^{-1}$ are reviewed, to see whether they give new indications of unknown or unsuspected prominent structures in the ZOA.

\section{Observational Surveys in Zone of Avoidance}

\subsection{Optical Surveys}

Systematic optical galaxy catalogues are generally limited to the largest galaxies (typically with diameters $D \gtrsim 1^{\prime}$, e.g. Lauberts 1982). These catalogues become, however, increasingly incomplete as the dust thickens, creating a 'Zone of Avoidance' in the distribution of galaxies of roughly $25 \%$ of the sky. Systematically deeper searches for partially obscured galaxies - down to fainter magnitudes and smaller dimensions compared to existing catalogues - were performed with the aim of reducing this ZOA. These surveys are not biased with respect to any particular morphological type.

Most of the ZOA has meanwhile been surveyed (cf. Figure 1 in Kraan-Korteweg 1998), revealing many galaxy overdensities uncorrelated with the patchy, optical extinction distribution. Analysing the galaxy density as a function of the galaxy size, magnitude and/or morphology in combination with the foreground extinction has led to the identification of various important large-scale structures and their approximate distances. Although the ZOA has been considerably reduced in this way (to about $10 \%$ of the sky), this method does not find galaxies in 

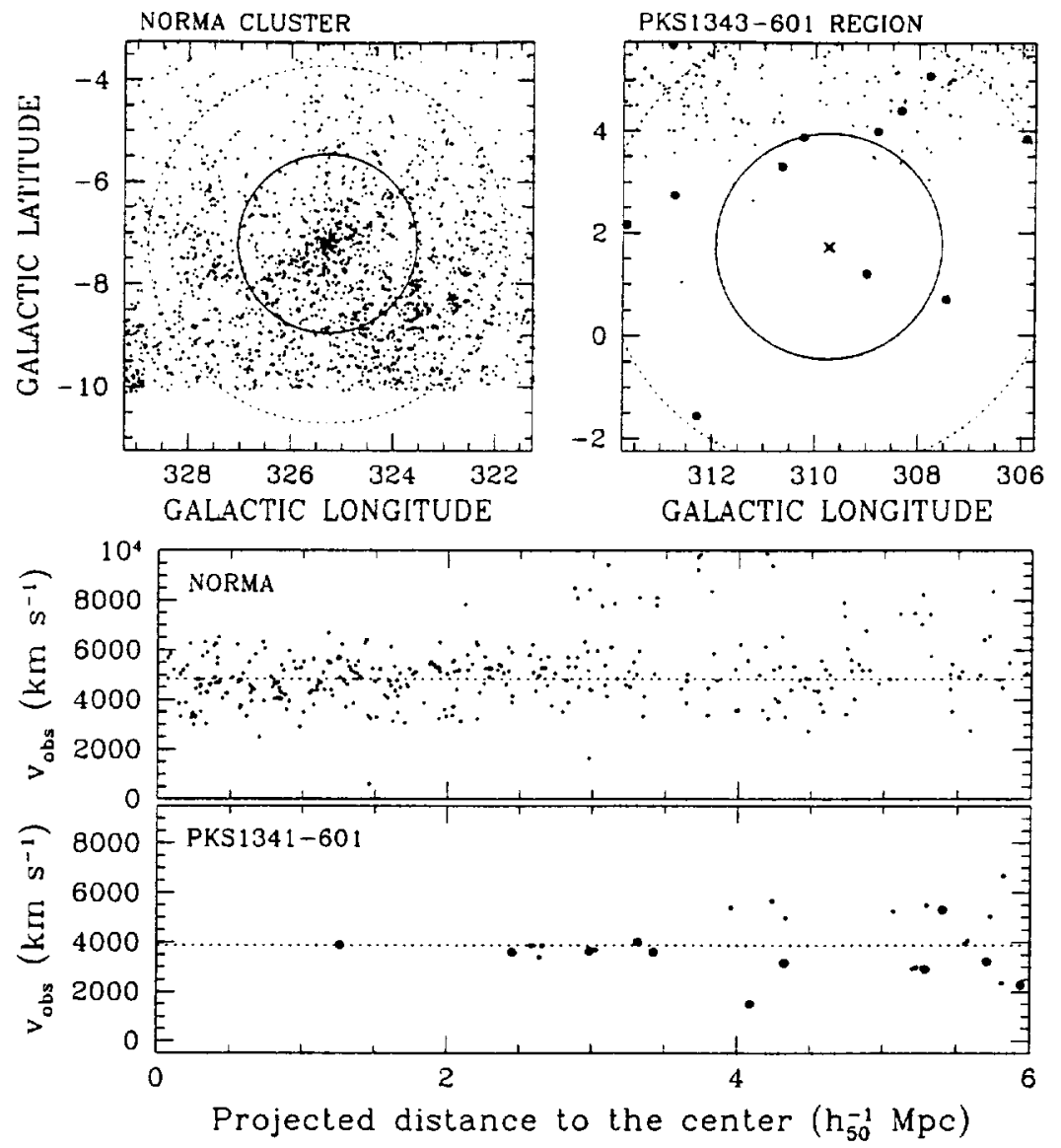

Figure 1-A comparison of the rich A3627 cluster $\left(A_{\mathrm{B}} \sim 1^{\mathrm{m}} .5\right)$ and the suspected PKS1343 cluster $\left(A_{\mathrm{B}} \sim 12^{\mathrm{m}}\right)$ in the GA region. Small dots are optically identified galaxies and large dots galaxies detected in the shallow Multibeam ZOA survey. The top panels display the sky distribution, where the inner circle marks the Abell radius $R_{\mathrm{A}}=3 h_{50}^{-1} \mathrm{Mpc}$, the bottom panel the redshift distribution as a function of distance to the central radio source.

the thickest extinction layers of the Milky Way, i.e. where the optical extinction exceeds 4-5 magnitudes at Galactic latitudes below $b \lesssim \pm 5^{\circ}$.

Redshift follow-ups of well-defined samples are important in mapping the large-scale structures in redshift space. So far, this has been pursued extensively in the Perseus-Pisces (PP) supercluster area and in large parts of the southern ZOA. The prominent new galaxy structures revealed in this way are summarised below. Their approximate positions (ordered in Galactic latitude) are given as $(\ell, b, v)$, with $v$ in units of $\mathrm{km} \mathrm{s}^{-1}$ :

- Behind the Galactic Bulge at $(0 \circ 5,9.5,8500)$, Wakamatsu et al. (1994) identified the rich Ophiuchus cluster (or supercluster) with some evidence of it being linked to the adjacent slightly more distant Hercules cluster.

- At $(\ell, b) \sim\left(33^{\circ}, 5^{\circ}-15^{\circ}\right)$, Marzke, Huchra \& Geller (1996) and Roman et al. (1998) found evidence for a nearby cluster close to the Local Void at $1500 \mathrm{~s}^{-1}$, as well as a prominent cluster behind the Local Void at $7500 \mathrm{~km} \mathrm{~s}^{-1}$. The nearby cluster is independently supported by data from the blind ZOA HI survey (Henning et al. 1999).
- The connection of the Perseus-Pisces supercluster across the ZOA to the cluster A569, suspected by Focardi, Marano \& Vettolani in 1984, was confirmed by Chamaraux et al. (1990) and Pantoja et al. (1997). The Perseus-Pisces chain folds back into the ZOA at higher redshifts at $\left(195^{\circ},-10^{\circ}, 7500\right)$, see Marzke, Huchra \& Geller (1996) and Pantoja et al. (1997).

- In 1992, Kraan-Korteweg \& Huchtmeier uncovered a nearby cluster in Puppis $\left(245^{\circ}, 0^{\circ}, 1500\right)$ which was later shown by Lahav et al. (1993) to contribute a not insignificant component to the peculiar $z$-motion of the Local Group.

- Kraan-Korteweg et al. (1994) presented evidence for a continous filamentary structure extending over $30^{\circ}$ on the sky from the Hydra and Antlia clusters across the ZOA, intersecting the Galactic Plane at $\left(280^{\circ}, 0^{\circ}, 3000\right)$. At the same longitudes, they noted significant clustering at $\sim 15,000 \mathrm{~s}^{-1}$, indicative of a connection between the Horologium and Shapley clusters a hundred degrees apart in the sky.

- Kraan-Korteweg \& Woudt (1993) uncovered a shallow but extended supercluster in Vela at $\left(285^{\circ}, 6^{\circ}, 6000\right)$. 
- Next to the massive cluster A3627 at the core of the Great Attractor (clustering in the Great Attractor region is discussed in the next section), Woudt (1998) discovered a cluster at $\left(306^{\circ}, 6^{\circ}, 6200\right)$ called the Cen-Crux cluster, and a more distant cluster, the Ara cluster at $\left(329^{\circ},-9^{\circ}, 15000\right)$. The latter might be connected to the Triangulum-Australis cluster.

2.1.1 Clustering within the Great Attractor Region Recent consensus is that the Great Attractor (GA) is probably an extended region $\left(\sim 40^{\circ} \times 40^{\circ}\right)$ of moderately enhanced galaxy density (Lynden-Bell 1991; Hudson 1994) centred behind the Galactic Plane at $(\ell, b, v) \sim\left(320^{\circ}, 0^{\circ}, 4500\right)$ (Kolatt, Dekel \& Lahav 1995).

Based on a deep optical galaxy search and subsequent redshift follow-ups, Kraan-Korteweg et al. (1996) and Woudt (1998) have clearly shown that the Norma cluster, A3627, at $\left(325.3,-7^{\circ} .2\right.$, 4882 ) is the most massive galaxy cluster in the GA region known to date and probably marks the previously unidentified but predicted density-peak at the bottom of the potential well of the GA overdensity. The prominence of this cluster has independently been confirmed by ROSAT observations: the Norma cluster ranks as the sixth brightest X-ray cluster in the sky (Böhringer et al. 1996). It is comparable in size, richness and mass to the well-known Coma cluster. Redshift-independent distance determinations $\left(\mathrm{R}_{\mathrm{C}}\right.$ and $\mathrm{I}_{\mathrm{C}}$ band Tully-Fisher analysis) of the Norma cluster have shown it to be at rest with respect to the rest frame of the Cosmic Microwave Background (Woudt 1998).

One cannot, however, exclude the possibility that other unknown rich clusters reside in the GA region. Finding a hitherto uncharted, rich cluster of galaxies at the heart of the GA would have serious implications for our current understanding of this massive overdensity in the local Universe. Woudt (1998) found various indications that PKS1343-601, the second brightest extragalactic radio source in the southern sky $\left(f_{20 \mathrm{~cm}}=79 \mathrm{Jy}\right.$; McAdam 1991, and references therein) might form the centre of yet another highly obscured rich cluster.

At $(\ell, b) \sim(309.7,1.7)$, this radio galaxy lies behind an obscuration layer of about 12 magnitudes of extinction in the B-band, as estimated from the DIRBE/IRAS extinction maps (Schlegel, Finkbeiner \& Davis 1998). Its observed diameter of 28 arcsec in the Gunn-z filter (West \& Tarenghi 1989) translates into an extinction-corrected diameter of 232 arcsec (following Cameron 1990). With a recession velocity of $v=3872 \mathrm{~km} \mathrm{~s}^{-1}$ (West \& Tarenghi 1989) this galaxy can be identified with a giant elliptical galaxy.

PKS1343-601 has recently been observed in the X-ray band with the ASCA satellite (Tashiro et al. 1998). This source is not detected in the ROSAT All Sky Survey due to the large foreground extinction, i.e. the soft X-ray emission is totally absorbed. However, extended diffuse hard X-ray emission at the position of PKS1343-601 has been detected with ASCA. The excess flux, $k T=3.9 \mathrm{keV}$ is far too large for it to be associated with a galactic halo surrounding the host galaxy, hence it might be due to the Inverse Compton process - or indicative of emission from a cluster.

As this prospective cluster is so heavily obscured, little data are available to substantiate the existence of this cluster. In Figure 1, a comparison of the A3627 cluster at $\left(325^{\circ} .3,-7.2,4882\right)$ and a mean extinction in the blue of $1^{\mathrm{m} .5}$ is compared to the prospective PKS1343 cluster at $(309 \circ 7,+1.7,3872)$ with an extinction of $12^{\mathrm{m}}$. The top panel shows both sky distributions. One can clearly see that at the low Galactic latitude of the suspected cluster PKS1343, the optical galaxy survey could not retrieve the underlying galaxy distribution, especially not within the Abell radius (the inner circle in the top right panel of Figure 1) of the suspected cluster. If PKS1343-601 marks the dynamical centre of the cluster, then the Abell radius, defined as $1.17 / z$, where $z$ is the redshift, corresponds to 2.2 on the sky at the redshift-distance of PKS1343-601.

Interestingly enough, the shallow blind ZOAMultibeam Hi survey (Henning et al. 1999) picks up a number of prospective cluster members even though the shallow survey is sensitive only to the most Hi-rich galaxies at the cluster velocity: over $60 \%$ of the galaxies in the shallow survey with velocities from 3000 to $5000 \mathrm{~km} \mathrm{~s}^{-1}$ lie in the cluster area, i.e. within $13 \%$ of the area covered by the shallow survey.

The velocity distribution as a function of distance from the cluster centre for the PKS1343-601 region (bottom panel) provides further evidence for the existence of this cluster. All measured velocities lie within a narrow range of the central radio source, showing a similar distribution as in the Norma cluster. One of the first data cubes from the full sensitivity Multibeam ZOA survey that has been finished covers the prospective PKS1343 cluster area. A quick inspection gives further support for this prospective cluster: between $3500<v<4000 \mathrm{~km} \mathrm{~s}^{-1}$ a statistically significant peak is evident in the velocity distribution (Juraszek et al. 1998, priv. commun.).

We will image the prospective cluster within its Abell radius in the near infrared (Woudt et al. 1999 in progress). These observations will allow us to determine whether or not PKS1343-601 is embedded in a centrally condensed overdensity of galaxies, comparable to the rich and massive Norma cluster.

\subsection{IRAS Surveys}

The IRAS Point-Source Catalogue (PSC) has been exploited in the last decade to identify galaxy 
candidates behind the ZOA. However, confusion with Galactic sources at low Galactic latitudes still leaves a considerable 'ZOA' of over $10 \%$. Moreover, bright spiral and starburst galaxies dominate these samples, and cluster cores are hardly visible.

The advantage of using the IRAS survey for LSS studies is the homogeneous sky coverage (all data from one instrument), and the various systematic redshift follow-ups, complete to given flux limits, i.e. 2658 galaxies to $f_{60 \mu \mathrm{m}}=1.9 \mathrm{Jy}$ (Strauss et al. 1992), 5321 galaxies to $f_{60 \mu \mathrm{m}}=1 \cdot 2 \mathrm{Jy}$ (Fisher et al. 1995), and $\sim 15000$ galaxies to $f_{60 \mu \mathrm{m}}=0 \cdot 6 \mathrm{Jy}$ (Saunders et al. 1999 in prep.).

Considerable improvement towards filling the ZOA has been made through the confirmation of about 1000 IRAS galaxy candidates in the ZOA from K-band snapshots (Saunders et al. 1999 in prep.).

Using the IRAS survey, dedicated searches for large-scale clustering within the whole ZOA $(|b| \leq$ $15^{\circ}$ ) have been made by Japanese groups (cf. Takata, Yamada \& Saito 1996, for a summary). They used IRAS colour criteria to select galaxy candidates which were subsequently verified through visual examination on sky surveys such as the Palomar Observatory Sky Survey (POSS) of the northern hemisphere and the ESO/SRC (United Kingdom Science Research Council) Southern Sky Atlas. Because of their verification procedure, this dataset suffers the same limitations in highly obscured regions as optical surveys.

Based on redshift follow-ups of this ZOA IRAS galaxy sample, they established various filamentary features and connections across the ZOA. Most coincide with the structures described in Section 2.1. Both crossings of the Perseus-Pisces arms into the ZOA are very prominent - considerably stronger in IRAS compared to optical data - and the Puppis, Hydra, Centaurus and A3627 connections are clearly visible. They furthermore identified a new structure: the Cygnus-Lyra filament at $\left(60^{\circ}-90^{\circ}, 0^{\circ}, 4000\right)$.

\subsection{HI Surveys}

In the regions of the highest obscuration and infrared confusion, the Galaxy is fully transparent to the 21-cm line radiation of neutral hydrogen. HI-rich galaxies can readily be found at lowest latitudes through the detection of their redshifted 21-cm emission. Early-type galaxies generally are very gas-poor and will not be uncovered in $\mathrm{HI}$ surveys. Furthermore, low-velocity extragalactic sources (blue- and red-shifted) within the strong Galactic Hi emission will be missed and-because of baseline ripple - galaxies close to radio continuum sources may also be missed.

As demonstrated by the first results from systematic Hi surveys (cf. Henning et al. 1999, Rivers et al. 1999 and Juraszek 1999-see pp. 35, 38 and 48), these surveys clearly are very powerful in tracing spiral and Hi-rich dwarf galaxies through the deepest extinction layer of the Milky Way. In particular, the results from the deep Multibeam ZOA survey will be very exciting as they will trace the galaxy distribution across the ZOA to a depth of $\sim 10,000$ $\mathrm{km} \mathrm{s}^{-1}$ (cf. Fig. 2 in Kraan-Korteweg et al. 1998).

\subsection{Near Infrared Surveys}

Near infrared (NIR) surveys are sensitive to earlytype galaxies, tracers of massive groups and clusters missed in IRAS and Hi surveys, and have little confusion with Galactic objects. Moreover, they are less affected by absorption than optical surveys. Here, the recent NIR surveys such as 2MASS (Skrutskie et al. 1997) and DENIS (Epchtein 1997) provide a new tool to probe the ZOA.

First results from DENIS data are very promising (cf. Schröder et al. 1999, this issue p. 42). They are complementary to other surveys in the sense that they finally uncover early-type galaxies at low Galactic latitudes $(|b| \gtrsim 1-1.5)$. Furthermore, a fair fraction $(\sim 65 \%)$ of the heavily obscured spiral galaxies detected in blind Hi surveys can be reidentified on DENIS images. The combination of $\mathrm{HI}_{\mathrm{I}}$ data with NIR data allows the study of the peculiar velocity field via the NIR Tully-Fisher relation 'in the ZOA' compared to earlier interpolations of data 'adjacent to the ZOA' and this will, for instance, provide important new input for density field reconstructions in the ZOA (cf. Section 3).

\subsection{X-ray Surveys}

The Milky Way is transparent to the hard X-ray emission, i.e. above $0 \cdot 5-2 \cdot 0 \mathrm{keV}$. Rich clusters generally are strong X-ray emitters. Hence, the X-ray surveys such as HEAO-1 and the ROSAT All Sky Survey provide an optimal tool to search for clusters of galaxies at low Galactic latitude. So far, this possibility has not yet been pursued in any systematic way, even though a large number of X-ray bright clusters (e.g. PKS0745-191) are located at low Galactic latitude (cf. Fabian 1994). This tool is of particular interest because it can unveil cores of clusters as, for instance, the suspected cluster surrounding PKS1343-601. These are dominated by early-type galaxies and therefore difficult to trace in other wavelengths.

\section{Theoretical Reconstructions}

Various mathematical methods exist to reconstruct the galaxy distribution in the ZOA without having access to direct observations. One possibility is the expansion of galaxy distributions adjacent to the ZOA into spherical harmonics to recover the structures in the ZOA, either with 2-dimensional catalogues (sky positions) or 3-dimensional data sets (redshift catalogues). 
A statistical method to reconstruct structures behind the Milky Way is the Wiener Filter (WF), developed explicitly for reconstructions of corrupt or incomplete data (cf. Lahav 1994; Hoffman 1994). Using the WF in combination with linear theory allows the determination of the real-space density of galaxies, as well as their velocity and potential fields.

The POTENT analysis developed by Bertschinger \& Dekel (1989) can reconstruct the potential field (mass distribution) from peculiar velocity fields in the ZOA (Kolatt, Dekel \& Lahav 1995). The reconstruction of the potential fields versus density fields have the advantage that they can locate hidden overdensities (their signature) even if 'unseen'.

Because of the sparsity of data and the heavy smoothing applied in all these methods, only structures on large scales (superclusters) can be mapped. Individual (massive) nearby galaxies that can perturb the dynamics of the Universe quite locally (the vicinity of the Local Group or its barycentre) will not be uncovered in this manner. But even if theoretical methods can outline LSS accurately, the observational efforts do not become superfluous. The comparison of the real galaxy distribution $\delta_{g}(\mathbf{r})$, from e.g. complete redshift surveys, with the peculiar velocity field $\mathbf{v}(\mathbf{r})$ will lead to an estimate of the density and biasing parameter $\left(\Omega^{0 \cdot 6} / b\right)$ through the equation

$$
\nabla \cdot \mathbf{v}(\mathbf{r})=-\frac{\Omega^{0 \cdot 6}}{b} \delta_{g}(\mathbf{r})
$$

cf. Strauss \& Willick (1995) for a detailed review.

\subsection{Early Predictions}

Early reconstructions on relatively sparse data galaxy catalogues have been performed within volumes out to $v \leq 5000 \mathrm{~km} \mathrm{~s}^{-1}$. Despite heavy smoothing, they have been quite successful in pinpointing a number of important features:

- Scharf et al. (1992) applied spherical harmonics to the 2-dimensional IRAS PSC and noted a prominent cluster behind the ZOA in Puppis $\left(\ell \sim 245^{\circ}\right)$ which was simultaneously discovered as a nearby cluster through Hi-observations of obscured galaxies in that region by Kraan-Korteweg \& Huchtmeier (1992).

- Hoffman (1994) predicted the Vela supercluster at $\left(280^{\circ}, 6^{\circ}, 6000\right)$ using 3 -dimensional WF reconstructions on the IRAS 1.9 Jy redshift catalogue (Strauss et al. 1992), which was observationally discovered just a bit earlier by Kraan-Korteweg \& Woudt (1993).

- Using POTENT analysis, Kolatt et al. (1995) predicted the centre of the Great Attractor overdensityits density peak-to lie behind the ZOA at $\left(320^{\circ}, 0^{\circ}, 4500\right)$. Shortly thereafter, Kraan-Korteweg et al. (1996) unveiled the cluster A3627 as being very rich and massive and at the correct distance. It hence is the most likely candidate for the central density peak of the GA.

\subsection{Deeper Reconstructions}

Recent reconstructions have been applied to denser galaxy samples covering larger volumes (8-10,000 $\mathrm{km} \mathrm{s}^{-1}$ ) with smoothing scales of the order of $500 \mathrm{~km}$ $\mathrm{s}^{-1}$ (compared to $1200 \mathrm{~km} \mathrm{~s}^{-1}$ ). It therefore seemed of interest to see whether these reconstructions find evidence for unknown major galaxy structures at higher redshifts.

The currently most densely-sampled, well-defined galaxy redshift catalogue is the Optical Redshift Survey (Santiago et al. 1995). However, this catalogue is limited to $|b| \geq 20^{\circ}$ and the reconstructions (cf. Baker et al. 1998) within the ZOA are strongly influenced by $1 \cdot 2$ Jy IRAS Redshift Survey data and a mock galaxy distribution in the inner ZOA. We will therefore concentrate on reconstructions based on the $1 \cdot 2$ Jy IRAS Redshift Survey only. In the following, the structures identified in the ZOA by (a) Webster, Lahav \& Fisher (1997, WLT) using WF plus spherical harmonics and linear theory and (b) Bistolas (1998) who applied a WF plus linear theory and non-constrained realisations on the $1 \cdot 2$ Jy IRAS Redshift Survey will be discussed and compared to observational data. Fig. 2 in Webster et al. displays the reconstructed density fields on shells of 2000, 4000, 6000 and $8000 \mathrm{~km} \mathrm{~s}^{-1}$; Fig. $5 \cdot 2$ in Bistolas displays the density fields in the ZOA from 1500 to $8000 \mathrm{~km} \mathrm{~s}^{-1}$ in steps of $500 \mathrm{~km}$ $\mathrm{s}^{-1}$.

The WLF reconstructions clearly find the recently identified nearby cluster at $\left(33^{\circ}, 5^{\circ}-15^{\circ}, 1500\right)$, whereas Bistolas reveals no clustering in the region of the Local Void out to $4000 \mathrm{~km} \mathrm{~s}^{-1}$. At the same longitudes, the clustering at $7500 \mathrm{~km} \mathrm{~s}^{-1}$ is seen by Bistolas, but not by Webster et al. The PerseusPisces chain is strong in both reconstructions, and the second Perseus-Pisces arm - which folds back at $\ell \sim 195^{\circ}$ - is clearly confirmed. Both reconstructions find the Perseus-Pisces complex to be very extended in space, i.e. from $3500 \mathrm{~km} \mathrm{~s}^{-1}$ out to $9000 \mathrm{~km}$ $\mathrm{s}^{-1}$. Whereas the GA region is more prominent compared to Perseus-Pisces in the Webster et al. reconstructions, the signal of the Perseus-Pisces complex is considerably stronger than the GA in Bistolas where it does not even reveal a well-defined central density peak. Both reconstructions find no evidence for the suspected PKS1343 cluster but its signal could be hidden in the central (A3627) density peak due to the smoothing. While the Cygnus-Lyra complex $\left(60^{\circ}-90^{\circ}, 0^{\circ}, 4000\right)$ discovered by Takata, Yamada \& Saito (1996) stands out clearly in Bistolas, it is not evident in Webster et al. Both reconstructions find a strong signal for the Vela SCL $\left(285^{\circ}, 6^{\circ}, 6000\right)$, 
labelled as HYD in WLF. The Cen-Crux cluster identified by Woudt (1998) is evident in Bistolas though less distinct in Webster et al. A suspected connection at $(\ell, v) \sim\left(345^{\circ}, 6000\right)$ - cf. Fig. 2 in Kraan-Korteweg, Koribalski \& Juraszek (1998)is supported by both methods. The Ophiuchus cluster just becomes visible in the most distant reconstruction shells $\left(8000 \mathrm{~km} \mathrm{~s}^{-1}\right)$.

\subsection{Conclusions}

Not all reconstructions find the same features, and when they do, the prominence of the density peaks as well as their locations in space do vary considerably. At velocities of $\sim 4000 \mathrm{~km} \mathrm{~s}^{-1}$ most of the dominant structures lie close to or within the ZOA while at larger distances, clusters and voids seem to be more homogeneously distributed over the whole sky. Out to $8000 \mathrm{~km} \mathrm{~s}^{-1}$ none of the reconstructions predict any major structures which are not mapped or suggested from observational data. So no major surprises seem to remain hidden in the ZOA. The various multi-wavelength explorations of the Milky Way will soon be able to verify this. Still, the combination of both the reconstructed potential fields and the observationally mapped galaxy distribution will lead to estimates of the cosmological parameters $\Omega$ and $b$.

\section{Acknowledgments}

The collaborations with our colleagues in the various multi-wavelength surveys - C. Balkowski, V. Cayatte, A. P. Fairall, with the redshift follow-ups of the optical surveys, A. Schröder and G. A. Mamon in the exploration of the DENIS survey, W. B. Burton, P. A. Henning, O. Lahav and A. Rivers in the northern ZOA Hi survey (DOGS) and the HIPASS ZOA team members R. D. Ekers, A. J. Green, R. F. Haynes, P. A. Henning, S. Juraszek, M. J. Kesteven, B. Koribalski, R. M. Price, E. Sadler and L. Staveley-Smith in the southern ZOA surveyare greatly appreciated. RKK warmly thanks the University of Western Sydney for partial financial suppport.

\section{References}

Baker, J. E., Davis, M., Strauss, M. A., et al. 1998, ApJ, 508, 6

Bertschinger, E., \& Dekel, A., 1989, ApJ, 336, 5

Bistolas, V. 1998, PhD thesis, Hebrew University, Jerusalem Böhringer, H., Neumann, D. M., Schindler, S., \& KraanKorteweg, R. C. 1996, ApJ, 467, 168

Cameron, L. M. 1990, A\&A, 233, 16

Chamaraux, P., Cayatte, V., Balkowski, C., \& Fontanelli, P. 1990, A\&A, 229, 340

Epchtein, N. 1997, in The Impact of Large Scale Near-Infrared Surveys, ed. F. Garzon et al. (Dordrecht: Kluwer), p. 15

Fabian, A. C. 1994, in Unveiling Large-Scale Structures behind the Milky Way, ed. C. Balkowski \& R. C. Kraan-
Korteweg, ASP Conf. Ser. 67 (San Franciso: ASP), p. 76

Fisher, K. B., Davis, M., Strauss, M. A., et al. 1995, ApJS, 100,69

Focardi, P., Marano, B., \& Vettolani, G. 1984, A\&A, 136, 178

Henning, P. A., Staveley-Smith, L., Kraan-Korteweg, R. C., \& Sadler, E. M. 1999, PASA, 16, 35

Hoffman, Y. 1994, in Unveiling Large-Scale Structures behind the Milky Way, ed. C. Balkowski \& R. C. Kraan-Korteweg, ASP Conf. Ser. 67 (San Franciso: ASP), p. 185

Hudson, M. 1994, MNRAS, 266, 475

Juraszek, S. 1999, PASA, 16, 38

Kolatt, T., Dekel, A., \& Lahav, O. 1995, MNRAS, 275, 797

Kraan-Korteweg, R. C. 1998 in The Low Surface Brightness Universe, IAU Coll 171, ed. J. I. Davies, C. Impey \& S. Philipps, ASP Conf. Ser. (San Franciso: ASP), in press (astro-ph/9810255)

Kraan-Korteweg, R. C., \& Huchtmeier, W. K. 1992, A\&A, 266, 150

Kraan-Korteweg, R. C., \& Woudt, P. A. 1993, in Cosmic Velocity Fields, ed. F. Bouchet \& M. Lachièze-Rey, Editions Frontières, p. 557

Kraan-Korteweg, R. C., Cayatte, V., Fairall, A. P., et al. 1994, in Unveiling Large-Scale Structures behind the Milky Way, ed. C. Balkowski \& R. C. Kraan-Korteweg, ASP Conf. Ser. 67 (San Franciso: ASP), p. 99

Kraan-Korteweg, R. C., Woudt, P. A., Cayatte, V., et al. 1996, Nature, 379, 519

Kraan-Korteweg, R. C., Koribalski, B., \& Juraszek, S. 1999, in Looking Deep in the Southern Sky, ed. R. Morganti \& W. Couch (New York: Springer), p. 23

Lahav, O. 1994, in Unveiling Large-Scale Structures behind the Milky Way, ed. C. Balkowski \& R. C. Kraan-Korteweg, ASP Conf. Ser. 67 (San Franciso: ASP), p. 171

Lahav, O., Yamada, T., Scharf, C. A., \& Kraan-Korteweg, R. C. 1993, MNRAS, 262, 711

Lauberts, A. 1982, The ESO/Uppsala Survey of the ESO (B) Atlas (Garching: ESO)

Lynden-Bell, D. 1991, in Observational Test of Cosmological Inflation, ed. T. Shanks et al. (Dordrecht: Kluwer), p. 337

McAdam, W. B. 1991, PASA, 9, 255

Marzke, R. O., Huchra, J. P., \& Geller, M. J. 1996, AJ, 112,1803

Pantoja, C. A., Altschuler, D. R., Giovanardi, C., \& Giovanelli, R. 1997, AJ, 113, 905

Rivers, A. J., Henning, P. A., \& Kraan-Korteweg, R. C. 1999, PASA, 16, 48

Roman, A. T., Takeuchi, T. T., Nakanishi, K., \& Saito, M. 1998, PASJ, 50, 47

Santiago, B. X., Strauss, M. A., Lahav, O., et al. 1995, ApJ, 446,457

Scharf, C., Hoffman, Y., Lahav, O., et al. 1992, MNRAS, 256,229

Schlegel, D. J., Finkbeiner, D. P., \& Davis, M. 1998, ApJ, 500,525

Schröder, A., Kraan-Korteweg, R. C., \& Mamon, G. A. 1999, PASA, 16, 42

Skrutskie, M. F., et al. 1997, in The Impact of Large Scale Near-Infrared Surveys, ed. F. Garzon et al. (Dordrecht: Kluwer), p. 25

Strauss, M. A., Huchra, J. P., Davis, M., et al. 1992, ApJS, 82,29

Strauss, M. A., \& Willick, J. A. 1995, Phys. Rep., 26, 27

Takata, T., Yamada, T., \& Saito, M. 1996, ApJ, 457, 693

Tashiro, M., Kaneda, H., Makishima, K., et al. 1998, ApJ, 499,713 
Wakamatsu, K., Hasegawa, T., Karoji, H., et al. 1994, in Unveiling Large-Scale Structures Behind the Milky Way, ed. C. Balkowski \& R. C. Kraan-Korteweg, ASP Conf. Ser. 67 (San Franciso: ASP), p. 131
Webster, M., Lahav, O., \& Fisher, K. 1997, MNRAS, 287, 425

West, R. M., \& Tarenghi, M. 1989, A\&A 223, 61 Woudt, P. A. 1998, PhD thesis, Univ. of Cape Town 